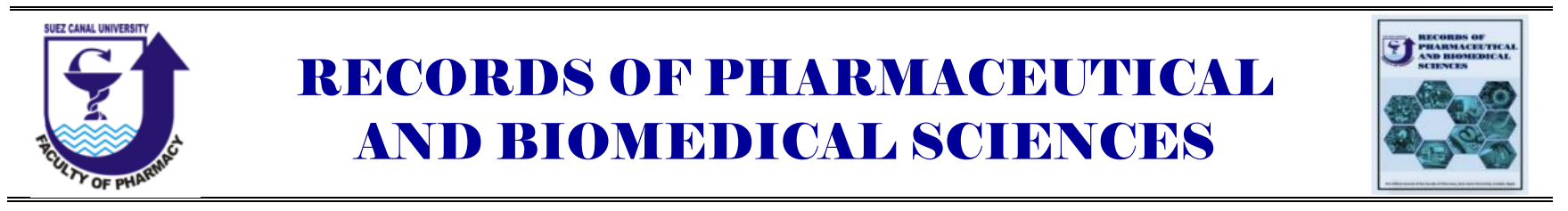

\title{
In-silico Screening of Selected Apiaceae Plants as Melanogenesis Inhibitors and their Predicted Skin Permeability
}

\author{
Reham S. Ibrahim* \\ Department of Pharmacognosy, Faculty of Pharmacy, Alexandria University, Alexandria 21521, Egypt.
}

Received on: 07. 01. 2020

Revised on: 28. 01. 2020

Accepted on: 08. 02. 2020

Correspondence Author:

Tel: $+20-01223821098$

E-mail address:

reham.abdelkader@alexu.edu.eg, rehamsaid84@yahoo.com

\begin{abstract}
Tyrosinase-related protein 1 (TYRP1) is a member of tyrosinase enzyme family which is metal-containing glycoproteins localized in melanosomes where the melanin biosynthesis occurs. The downregulation of tyrosinase is the most protruding approach for the development of skin whitening and depigmentation agents, however, TYRP1 downregulation is considered a novel target for melanogenesis inhibition and is still under clinical investigations for treatment of malignant melanoma. In the present study, virtual screening using glide extra-precision docking of an in-house generated data base of 400 natural compounds belonging to family Apiaceae for TYRP1 inhibitors was performed for the first time. luteolin-7-apiosylglucoside from celery followed by 4-( $\beta$-D-glucopyranosyloxy)benzoic acid then caffeoylquininc acid from anise showed highly potent melanogenesis inhibition even more than the synthetic standard inhibitors such as azelaic and kojic acids. These natural inhibitors exhibited good predicted skin permeability after processing with Qikprop module. Although they had slow predicted transdermal penetration rates, but these rates can be enhanced through optimizing their topical pharmaceutical formulations.
\end{abstract}

Keywords: human tyrosinase -related protein 1; Apiaceae; docking; skin permeability; melanogenesis.

\section{Introduction}

Melanogenesis is a process of synthesis of melanin, which is chiefly responsible for human skin, eye and hair pigmentation. However, over production of melanin may results in hyperpigmentation malfunctions like melanoma and freckles (Briganti et al., 2003). In humans, three enzymes are involved in the biosynthesis of melanin; the first enzyme is tyrosinase (TYR) which is the critical and rate-limiting enzyme; it catalyzes tyrosine oxidation that is considered the rate limiting step of the melanin synthesis
(Halaban et al., 2002). The second enzyme is tyrosinase-related protein 2 (TYRP2) which is a tautomerase enzyme and the third one is tyrosinaserelated protein 1 (TYRP1) which was found to be overexpressed in melanocytes in case of malignant melanoma. All these tyrosinase enzyme family members are metal-containing glycoproteins localized in melanosomes where the melanin biosynthesis occurs (Lai et al., 2017). The downregulation of TYR is the most protruding approach for the development of skin whitening 


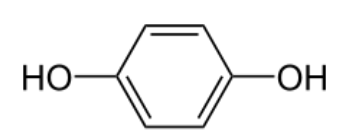

Hydroquinone<smiles>OCC1C(O)C(O)C(O)C(Oc2ccc(O)cc2)C1O</smiles>

Arbutin<smiles>O=C(O)CCCCCCCC(=O)O</smiles>

Azelaic acid<smiles>O=c1cc(CO)occ1O</smiles>

Kojic acid

Figure 1: Structure of standard melanogenesis inhibitors.

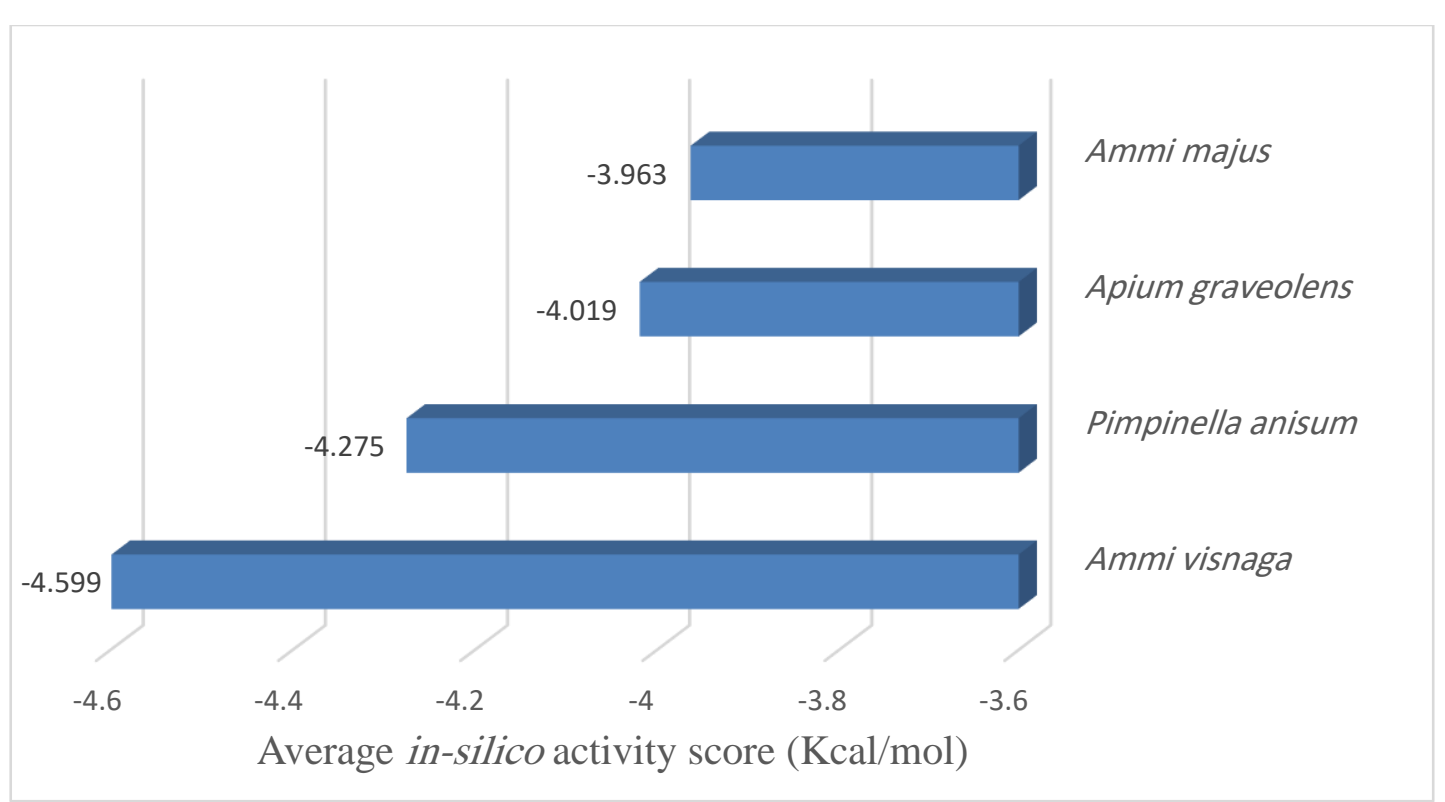

Figure 2: Average in-silico activity scores (Kcal/mol) of the selected Apiaceae plant species.

Table 1: Extra-precision Glide docking with TYRP1 and PyRx docking scores with human TYR (Kcal/mol) for standard melanogenesis inhibitors:

\begin{tabular}{|c|c|c|c|c|}
\hline $\begin{array}{c}\text { Docking scores } \\
\text { (Kcal/mol) }\end{array}$ & Azelaic acid & Kojic acid & Arbutin & Hydroquinone \\
\hline TYRP1 XP G score & -9.793 & -7.719 & -4.621 & -3.428 \\
\hline TYR PyRx score* & -2.57 & -4.21 & -4.62 & -4.07 \\
\hline
\end{tabular}

\footnotetext{
* the docking results of human TYR were adopted from the study performed by (Hassan et al., 2018)
} 
and depigmentation agents (Pillaiyar et al., 2017), however, TYRP1 downregulation is considered a novel target for melanogenesis inhibition and still under clinical investigations (Khalil et al., 2016). Various synthetic melanogenesis inhibitors have been launched to the market such as hydroquinone, azelaic acid, kojic acid and arbutin (Fig. 1). However, these synthetic depigmentation agents are greatly associated with health concerns. For example; hydroquinone is mutagenic and possesses many side effects such as contact dermatitis, leukoderma, burning and irritating sensation (Curto et al., 1999). Azelaic acid, although demonstrated no systemic adverse effects, but it showed cutaneous erythema, scaling and pruritus (Fitton and Goa, 1991). The use of kojic acid as skin whitening agent has been restricted because of its carcinogenicity and instability (Gonçalez et al., 2013). Arbutin, is a natural depigmenting agent. However, arbutin in its natural forms is unstable and can release hydroquinone which is metabolized to benzene with the prospective bone marrow toxicity (Zhou et al., 2009). For these reasons, a great interest in recent years to find natural, safer and effective alternatives for synthetic depigmenting agents has been evolved (Gholamhoseinian and Razmi, 2012).

In-vitro screening of medicinal plants to discover new melanogenesis inhibitors was performed (Gholamhoseinian and Razmi, 2012), However, in-vitro approaches have drawbacks of being expensive and time-consuming, especially when large number of compounds needed to be screened as in case of phytoconstituents present in medicinal plants (Lionta et al., 2014). Hence, insilico screening utilizing computational speed, being fast and cost-effective, possesses great advantages in analyzing large number of compounds for discovering new drugs with good pharmacokinetic potential (Shen et al., 2003).

Recently, few coumarins such as esculetin and umbelliferone have been proved to possess strong TYR inhibitory activity (Masamoto et al., 2004)(Ashraf et al., 2015). Moreover, many phenols, phenolic acids and flavonoids were identified as TYR blockers and hence skin whiteners (Lin et al., 2008). Family Apiaceae is considered as a plentiful source of these phytoconstituents. In this context, and by applying multi-targeting approach, four plant species rich in TYR inhibitors belonging to family Apiaceae were selected for screening of their constituents as
TYRP1 inhibitors in this work. By this dual target we can achieve more efficient melanogenesis inhibition. In the present study, a huge 3D in-house data base of natural compounds from the selected Apiaceae plants were constructed. In-silico screening of this data base for their TYRP1 inhibitory activity was performed. The virtual activity and predicted skin permeability of the top natural compounds were compared for the first time to that of the standard synthetic skin depigmenting agents aiming at discovering novel, effective and safe melanogenesis inhibitors.

\section{Materials and Methods}

\subsection{Criteria of selection of candidate plants from family Apiaceae:}

Four plant species belonging to family Apiaceae namely: Pimpinella anisum, Apium graveolens, Ammi visnaga and Ammi majus fruits were selected for screening as TYRP1 inhibitors. These species were selected as they represent a rich source of coumarins, phenolic acids and flavones with potential TYR inhibitory activity (Lin et al., 2008). Prenyloxycoumarins have been revealed as one of the most promising skin whitening compounds (Taddeo et al., 2019). Ammi (Abu-Mustafa et al., 1971), and Apium (Shakeri et al., 2014) species represent main natural sources of these prenyloxycoumarins.

\subsection{D-in-house data base generation from candidate plants' phytoconstituents}

A database was compiled from 400 natural compounds representing the phytoconstituents of the four selected plant species. These compounds were imported as (sdf) files from our previous work (Ibrahim et al, 2020) .

\subsection{In-silico screening via molecular docking:}

The X-ray crystal structure of human TYRP1 (PDB: 5M8N) co-crystallized with kojic acid was downloaded from RCBS Protein Data Bank (http://www.pdb.org). This tyrosinase enzyme crystal structure was chosen based on its high resolution $(2.65 \AA)$, in addition, it was recently released in 2017. The protein was prepared using Schrödinger ${ }^{\circledR}$ suite 2015-2 software (Schrödinger, 2015, LLC, New York, NY) where hydrogens were added and the water molecules beyond $5 \AA$ were deleted. The Protein structures were reviewed and modified followed by assignment of hydrogen 
bonds using PROPKA at $\mathrm{PH}=7$. Finally, the energy was minimized using OPLS3 force field.

\subsubsection{Preparation of ligands:}

Ligands' structures were prepared using the LigPrep module of Schrödinger. Several poses for each input structure were produced to achieve their corresponding low-energy conformers using OPLS3 force field. The ionization states of the ligands were generated using Epik function at the physiological $\mathrm{pH}$ and tautomers were generated for each state.

\subsubsection{Grid generation and molecular docking:}

Receptor grid where the docking process takes place was generated with the GLIDE Grid generation module. Boxes enclosing the centroids of co-crystallized ligand (Kojic acid) were selected. GLIDE extra precision (XP) docking protocol was selected and performed in DELL system with Intel Core $\mathrm{i} 7$ and Windows 10 as an operating system.

\subsection{Prediction of transdermal penetration:}

The predicted skin permeability of the top-scored compounds was evaluated using QikProp module imbedded in the Schrödinger suite. Prediction of transdermal penetration was conducted through computing the predicted skin permeability $(\operatorname{logKp})$ and the maximum transdermal transport rates $(\mathrm{Jm})$ values.

\section{Results and Discussion}

Plant species belonging to family Apiaceae have recently been established to biosynthesize prenyloxycoumarins; a wide series of oxyprenylated secondary metabolites, in particular umbelliferone derivatives (Taddeo et al., 2017). These oxyprenylated coumarins in addition to other coumarins, flavonoids and phenolic acids are considered to be strong TYR inhibitors (Lin et al., 2008). For this reason, we selected four Apiaceae species; Apium graveolens, P Pimpinella anisum, Ammi visnaga and Ammi majus for in-silico screening for novel phytoconstituents with TYRP1 inhibitory activity.

\subsection{Ranking of the selected plant species based on their average in-silico activity scores:}

Molecular docking of the 3D in-house database compiled of 400 compounds into the crystalline structure of human TYRP1 (PDB: 5M8N) yielded 328 active compounds. An average in-silico TYRP1 inhibitory activity score for each plant species was calculated by summing the docking scores (Kcal/mol) of their constituents and dividing the sum by the total number of its constituents in the original data base (Dawood et al., 2018). Fig. 2 shows the ranking of the selected plant species based on their average in-silico activity scores. It was found that Ammi visnaga exhibited the strongest TYRP1 inhibitor with average score ($4.599 \mathrm{Kcal} / \mathrm{mol}$ ), followed by Pimpinella anisum, while, Apium graveolens and Ammi majus exhibited the lowest activity. The high activity of A. visnaga was confirmed by the study formed by Ashour et al. which demonstrated that constituents from A. visnaga such as khellin and khellol inhibited melanin biosynthesis (Ashour et al., 2013). These findings suggest $A$. visnaga extract as a promising candidate to be included in skin whitening formulations after further in-vitro and invivo testing.

\subsection{Docking interactions with human TYRPI active site:}

The typical tyrosinase-like subdomain of TYRP1 glycoenzyme contains two zinc ions in the active site instead of copper ions as found in tyrosinases. the specific melanogenic function of TRP1 is the oxidation of 5,6-dihydroxyindole-2-carboxylic acid to a carboxylated indole-quinone at a downstream point in the melanin biosynthetic pathway (Kobayashi et al., 1994). TYRP1 contains 4 conserved regions: the signal peptide, the intramelanosomal domain that is subdivided into the Cys-rich and the tyrosinase-like subdomains, the transmembrane $\alpha$-helix, and the cytoplasmic domain (Lai et al., 2017).

\subsubsection{Interactions of the standard melanogenesis inhibitors with TYRP1:}

Extra-precision docking of the standard skin whitening agents (Fig.1) within the active site of TYPP1 revealed that azelaic acid with docking score $-9.793 \mathrm{Kcal} / \mathrm{mol}$ exhibited the highest inhibitory activity followed by kojic acid then arbutin and finally hydroquinone (Table 1). Although hydroquinone is a potential human TYR inhibitor, it showed a weak TYRP1 activity. Azelaic and kojic acids experienced a dual inhibitory activity on both melanogenesis targets as shown from docking scores in Table 1. Hence, they were selected for further study of their detailed interactions within the active site of TYRP1. By observing the 2D and 3D interaction diagrams of TYRP1 with azelaic and kojic acids 

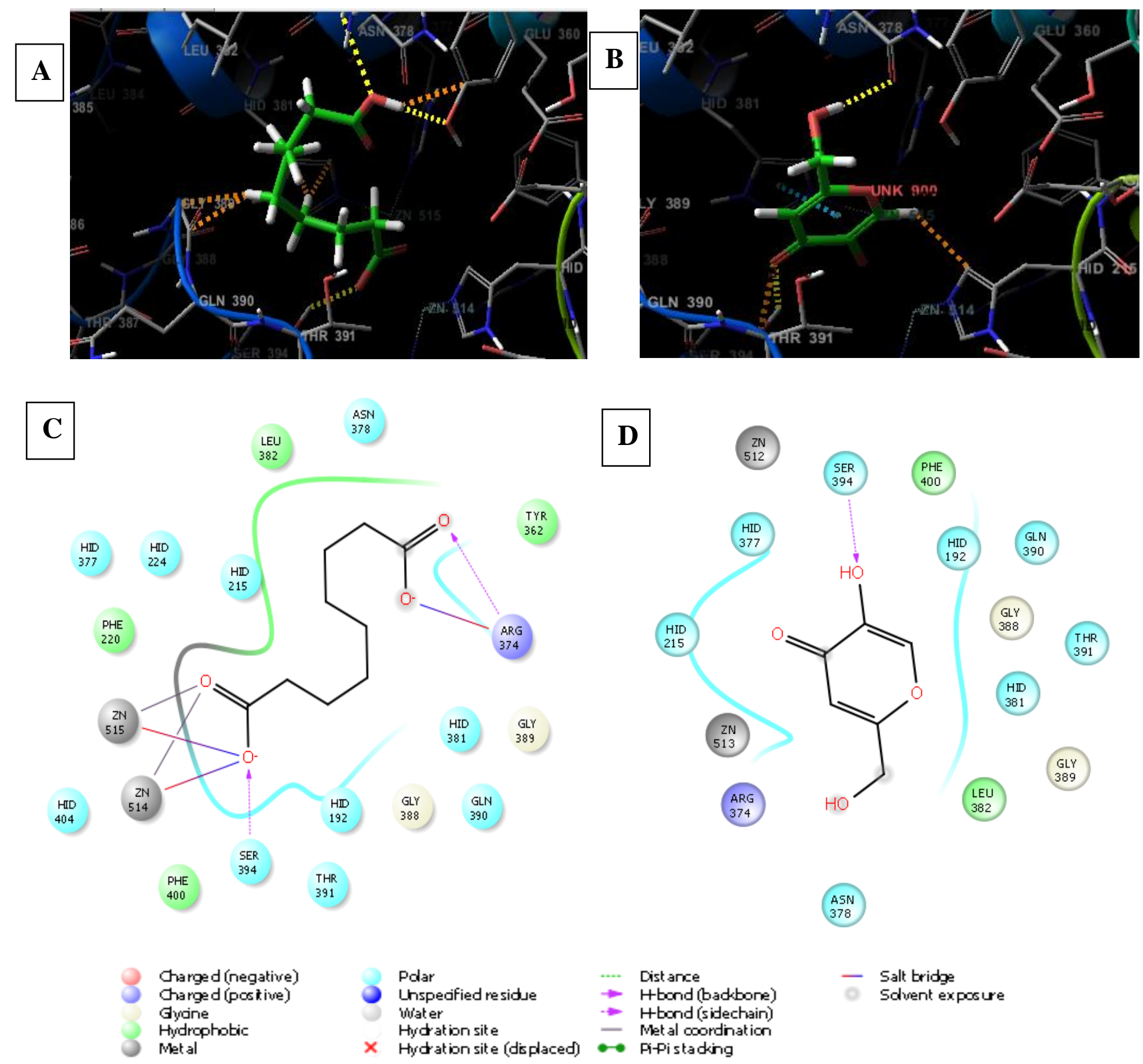

Figure 3: 2D and 3D Interaction diagrams of TYRP1 active site with azelaic acid (A and C, respectively) and with kojic acid ( $B$ and $D$, respectively). 
Table 2: Types of binding interactions between and luteolin-7-apiosylglucoside, 4- $(\beta$-Dglucopyranosyloxy)benzoic acid and caffeoylquininc acid and the important amino acid residues in the binding site of TYRP1 together with their docking scores:

\begin{tabular}{|c|c|c|c|}
\hline Ligand & $\begin{array}{l}\text { Docking XP G } \\
\text { scores } \\
\text { (Kcal/mol) }\end{array}$ & $\begin{array}{c}\text { Binding amino acid residues in } \\
\text { TYRP1 active site }\end{array}$ & Type of interaction \\
\hline \multirow{4}{*}{$\begin{array}{l}\text { luteolin-7- } \\
\text { apiosylglucoside }\end{array}$} & \multirow{4}{*}{-11.6} & $\begin{array}{l}\text { Asp212, Glu216, Asn318, Arg374, } \\
\text { Asn378, Leu382, Gln390, Thr391 }\end{array}$ & $\begin{array}{l}\text { Hydrogen bonding with } \\
\text { backbone and side chains }\end{array}$ \\
\hline & & Asp212, Glu216 & $\begin{array}{lll}\text { Charged } & \text { (negative) ionic } \\
\text { interaction } & & \end{array}$ \\
\hline & & $\begin{array}{l}\text { Hid215, Asn318, Hid377, Asn378, } \\
\text { Hid381, Asn385, Gln 390, Thr391 }\end{array}$ & Polar interaction \\
\hline & & $\begin{array}{l}\text { Val196, Val211, Val324, Thy62, } \\
\text { Leu382 }\end{array}$ & Hydrophobic interaction \\
\hline \multirow{5}{*}{$\begin{array}{l}\text { 4-( } \beta \text {-D-glucopyran- } \\
\text { osyloxy) benzoic acid }\end{array}$} & \multirow{5}{*}{-11.1} & Tyr362, Arg374, Thr391, Ser394 & Hydrogen bonding interaction \\
\hline & & Zn514, Zn515 & $\begin{array}{l}\text { Metal coordinate bonding with } \\
\text { Zn }\end{array}$ \\
\hline & & $\begin{array}{lll}\text { Hid192, } 215, \quad 224, \quad 377, \quad 381, \\
\text { Asn378, Gln390, Thy391,Ser394, } \\
\text { Hid404 }\end{array}$ & Polar interaction \\
\hline & & Phe220, Tyr362, Leu382, Phe400 & Hydrophobic interaction \\
\hline & & Hid337, Hid381 & Pi-Pi stacking interaction \\
\hline \multirow{5}{*}{ caffeoylquininc acid } & \multirow{5}{*}{-10.8} & Val196, Tyr362, Thr391, Ser394 & Hydrogen bonding interaction \\
\hline & & Zn514, Zn515 & $\begin{array}{l}\text { Metal coordinate bonding with } \\
\text { Zn }\end{array}$ \\
\hline & & $\begin{array}{l}\text { Hid192, 215, 224, 377, 381, 404, } \\
\text { Asn378, Thr391, Ser394 }\end{array}$ & Polar interaction \\
\hline & & Val196, 211, Tyr362, Leu382 & Hydrophobic interaction \\
\hline & & Glu210, 216, 360, Asp212 & $\begin{array}{l}\text { Charged (negative) } \\
\text { interaction }\end{array}$ \\
\hline
\end{tabular}


(Fig.3), it was found that these standard melanogenesis inhibitors were successfully docked within the active site of the novel target enzyme. That was evidenced by the presence of the two zinc atoms which are essential for the activity (Lai et al., 2017). Kojic acid (Fig.3 B and D); the cocrystallized ligand, formed hydrogen bond with Ser394 residue and numerous polar interactions with Hid192, 215, 377, 381 (Histidine with hydrogen on the delta nitrogen), in addition to hydrophobic interactions with Phe400 and Leu382.

Azelaic acid (Fig.3 A and C) exhibited more interactions with TYRP1 than kojic acid and that explained its higher binding energy (Table 1). It formed four metal coordinate bonds with the binuclear zinc center, hydrogen bond with Arg374 and Ser394, many polar interactions with Hid192, 215, 224, 377, 381, 404, Asm378, Gln390, Thr391 and Van der Waals forces with Phe220, 400, Leu362 and 382 amino acid residues.

\subsubsection{Virtual screening of the 3D-data base of natural compounds:}

After structure-based virtual screening of the compiled database of natural products into the generated grid of TYRP1 crystal structure, the phytoconstituent were ranked according to their activity (docking scores) in a descending order. Figure 4 represents the docking XP G scores (Kcal/mol) of the top-scored 50 natural TYRP1 inhibitors from family Apiaceae with the standard melanogenesis inhibitors; azelaic and kojic acids. It was astonishing to observe that six natural compounds exceeded azelaic acid in TYRP1 inhibitory activity. These compounds were: luteolin-7-apiosylglucoside from celery followed by 4-( $\beta$-D-glucopyranosyloxy)benzoic acid then caffeoylquininc acid from anise, shikimic acid from celery, 3,3',4',5,7-pentahydroxyflavone-3-sulfate from $A$. visnaga and isochlorogenic acid from celery, with docking scores; -11.6, -11.1, -10.8, $10.3,-10.2$ and -10.1 (Kcal/mol), respectively. It was also observed that most of the top hits of TYRP1 inhibitors were phenolic acids rather than flavonoids and coumarins (Fig. 4) unlike TYR inhibitors from which coumarins were more predominant (Ashraf et al., 2015) (Masamoto et al., 2004).

The three top-scored hits of phytoconstituents were selected for further investigation of their interactions with TYRP1 active site (Figure 5). Table 2 summarizes the types of significant binding interactions between the important amino acid residues in the binding site and luteolin-7apiosylglucoside, 4-( $\beta$-D-glucopyranosyloxy) benzoic acid and caffeoylquininc acid.

\subsection{In-silico prediction of transdermal penetration:}

Computer-based techniques have been implemented in the prediction of pharmacokinetic properties of new drugs at an early stage of drug discovery. These in-silico approaches are becoming increasingly widespread (Lipinski et al., 1997). The main reason behind these approaches is the relatively lower cost and time needed, when compared to experimental ex-silico approaches for ADME (absorption, distribution, metabolism and excretion) profiling (Darvas et al., 2002).

Transdermal penetration is a crucial criterion when searching for new melanogenesis inhibitors that are intended to be administered through skin. In-silico prediction of transdermal penetration of the standard melanogenesis inhibitors in addition to the six compounds exceeding azelaic acid in TYRP1 inhibitory activity was conducted using QikProp module imbedded in the Schrödinger suite. Their transdermal penetration was evaluated through computing the predicted skin permeability (log Kp) and the maximum transdermal transport rates $(\mathrm{Jm})$ values (Schrödinger, 2007) as shown in Table 3. The recommended range of the predicted skin permeability is from -8 to -1 for $95 \%$ of all drugs (Potts and Guy, 1992). Upon investigating the values of $\log \mathrm{Kp}$ in Table 3, it was found that all the tested and standard compounds fall in the recommended range of $95 \%$ of known drugs indicating that all of them are skin permeable.

The predicted maximum transdermal transport rates, $\mathrm{Jm}$ in $\left(\mu \mathrm{g} / \mathrm{cm}^{2} / \mathrm{hr}\right)$ were computed from the aqueous solubility (Swat) and skin permeability $(\mathrm{Kp})$, using the following relation (Potts and Guy, 1992):

$\mathrm{Jm}=\mathrm{Kp} \times \mathrm{MW} \times \mathrm{Swat}$

This parameter (Table 3) showed variations from 0.0002 to $64.7 \mu \mathrm{g} / \mathrm{cm}^{2} / \mathrm{hr}$. Hydroquinone had the highest predicted value of $\mathrm{Jm}>64 \mu \mathrm{g} / \mathrm{cm}^{2} / \mathrm{hr}$. It was also observed that all the natural compounds had a very slow predicted transdermal transport rates compared to synthetic inhibitors which may be attributed to their high molecular weights. This slow penetration rate can be enhanced by optimizing their pharmaceutical formulations 


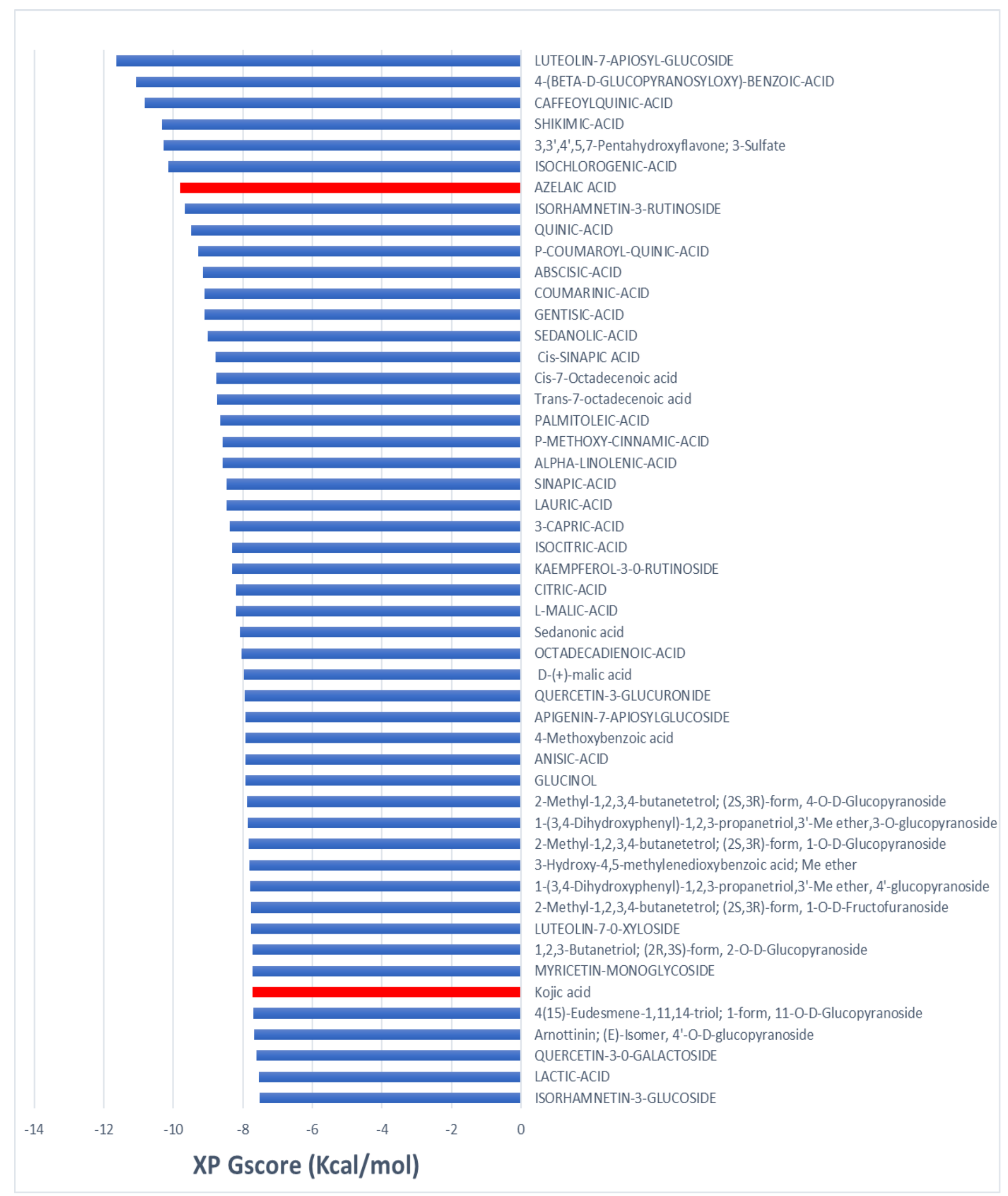

Figure 4: Docking XP G scores (Kcal/mol) of the top-scored 50 natural TYRP1 inhibitors from family Apiaceae with the standard melanogenesis inhibitors; azelaic and kojic acids. 

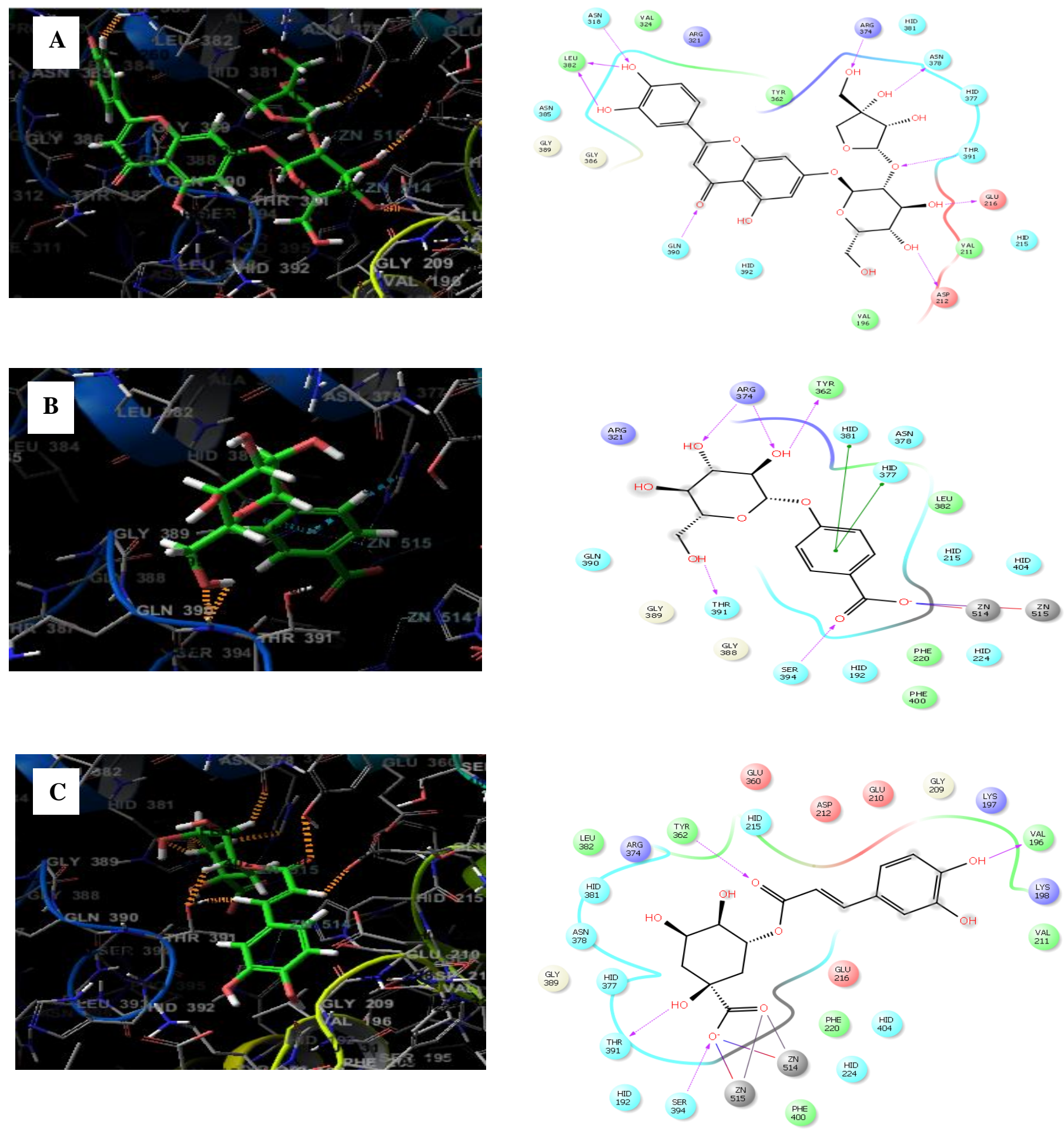

Gharged (negative)
Gharged (posive)
Gyaine
Hydrophotic
Met al

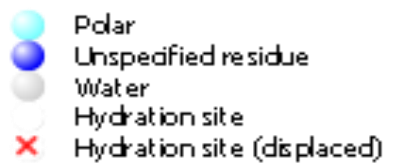

-... Dist ance
- Hbond (badkbone)
- Mbond (sidechain)
- Metal ooordination

- Salt bridge

Solvent exposure

Figure 5: 2D (to the right) and 3D (to the left) interaction diagrams of TYRP1 active site with the top three natural compound; luteolin-7-apiosylglucoside $(A), 4-(\beta$-Dglucopyranosyloxy)benzoic acid (B) and caffeoylquininc acid (C). 
Table 3: In-silico Qikprop transdermarmal penetration parameters of standard melanogenesis inhibitors in addition to top-scored six natural compounds

\begin{tabular}{|c|c|c|c|}
\hline Melanogenesis inhibitor & Molecular weight & QP log Kp & Jm \\
\hline Kojic acid & 142.111 & -3.900 & 5.1628 \\
\hline Hydroquinone & 110.112 & -2.703 & 64.7311 \\
\hline Azelaic acid & 188.223 & -4.714 & 0.0413 \\
\hline Arbutin & 272.254 & -4.506 & 0.3660 \\
\hline Luteolin-7-apiosylglucoside & 580.498 & -6.999 & 0.0002 \\
\hline 4-(Beta-D-glucopyranosyloxy)benzoic acid & 300.265 & -5.504 & 0.0262 \\
\hline Caffeoylquininc acid & 354.313 & -6.271 & 0.0005 \\
\hline Shikimic acid & 174.153 & -5.350 & 0.0847 \\
\hline 3,3',4',5,7-Pentahydroxyflavone-3-Sulfate & 382.298 & -6.667 & 0.0002 \\
\hline Isochlorogenic acid & 354.313 & -6.281 & 0.0005 \\
\hline
\end{tabular}

through incorporating skin penetration enhancers. All these in-silico skin permeation parameters were virtually predicted and are still needing further invitro testing to confirm them.

\section{Conclusion:}

The 3D-database of 400 natural phytoconstituents obtained from selected plants belonging to family Apiaceae included promising tyrosinase-related protein 1 (TYRP1) inhibitors. The inhibition of this target which possesses an integral role in melanogenesis inhibition was investigated for the first time in the present study. luteolin-7-apiosylglucoside from celery followed by

\section{References:}

Abu-Mustafa, E.A., El-Bay, F.K.A., Fayez, M.B.E., 1971. Natural coumarins XII: Umbelliprenin, a constituent of ammi majus L. fruits. J. Pharm. Sci. 60, 788-789.

Ashour, A., El-Sharkawy, S., Amer, M., Bar, F.A., Kondo, R., Shimizu, K., 2013. Melanin biosynthesis inhibitory activity of compounds isolated from unused parts of Ammi visinaga. J. Cosmet. Dermatological Sci. Appl. 3, 40.

Ashraf, Z., Rafiq, M., Seo, S.-Y., Babar, M.M., Zaidi, N.-S.S., 2015. Design, synthesis and bioevaluation of novel umbelliferone analogues as potential mushroom tyrosinase inhibitors. J. Enzyme Inhib. Med. Chem. 30, 874-883.

Briganti, S., Camera, E., Picardo, M., 2003. Chemical and instrumental approaches to treat hyperpigmentation. Pigment cell Res. 16, 101-110.

\section{4-( $\beta$-D-glucopyranosyloxy)benzoic acid}

then caffeoylquininc acid from anise, shikimic acid from celery, 3,3',4',5,7-pentahydroxyflavone-3sulfate from $A$. visnaga and isochlorogenic acid from celery showed highly potent inhibition as revealed from glide extra-precision molecular docking even more than azelaic and kojic acids. These natural inhibitors exhibited good predicted skin permeability after processing with QikProp module. Although these natural melanogenesis inhibitors had slow predicted transdermal penetration rates, but these rates can be enhanced through optimizing their topical pharmaceutical formulations.

Curto, E. V, Kwong, C., Hermersdörfer, H., Glatt, H., Santis, C., Virador, V., Hearing Jr, V.J., Dooley, T.P., 1999. Inhibitors of mammalian melanocyte tyrosinase: in vitro comparisons of alkyl esters of gentisic acid with other putative inhibitors. Biochem. Pharmacol. 57, 663-672.

Darvas, F., Keseru, G., Papp, A., Dorman, G., Urge, L., Krajcsi, P., 2002. In silico and ex silico ADME approaches for drug discovery. Curr. Top. Med. Chem. 2, 1287-1304.

Dawood, H.M., Ibrahim, R.S., Shawky, E., Hammoda, H.M., Metwally, A.M., 2018. Integrated in silico-in vitro strategy for screening of some traditional Egyptian plants for human aromatase inhibitors. J. Ethnopharmacol. 224. https://doi.org/10.1016/j.jep.2018.06.009

Fitton, A., Goa, K.L., 1991. Azelaic acid. Drugs 
41, 780-798.

Gholamhoseinian, A., Razmi, Z., 2012. Screening the methanolic extracts of some plants for tyrosinase inhibitory activity. Toxicol. Environ. Chem. 94, 310-318.

Gonçalez, M.L., Correa, M.A., Chorilli, M., 2013. Skin delivery of kojic acid-loaded nanotechnologybased drug delivery systems for the treatment of skin aging. Biomed Res. Int. 2013.

Halaban, R., Patton, R.S., Cheng, E., Svedine, S., Trombetta, E.S., Wahl, M.L., Ariyan, S., Hebert, D.N., 2002. Abnormal acidification of melanoma cells induces tyrosinase retention in the early secretory pathway. J. Biol. Chem. 277, 1482114828.

Khalil, D.N., Postow, M.A., Ibrahim, N., Ludwig, D.L., Cosaert, J., Kambhampati, S.R.P., Tang, S., Grebennik, D., Kauh, J.S.W., Lenz, H.-J., 2016. An open-label, dose-escalation Phase I study of antiTYRP1 monoclonal antibody IMC-20D7S for patients with relapsed or refractory melanoma. Clin. Cancer Res. 22, 5204-5210.

Kobayashi, T., Urabe, K., Winder, A., JiménezCervantes, C., Imokawa, G., Brewington, T., Solano, F., García-Borrón, J.C., Hearing, V.J., 1994. Tyrosinase related protein 1 (TRP1) functions as a DHICA oxidase in melanin biosynthesis. EMBO J. 13, 5818-5825.

Lai, X., Wichers, H.J., Soler-Lopez, M., Dijkstra, B.W., 2017. Structure of human tyrosinase related protein 1 reveals a binuclear zinc active site important for melanogenesis. Angew. Chemie Int. Ed. 56, 9812-9815.

Lin, J.-W., Chiang, H.-M., Lin, Y.-C., Wen, K.-C., 2008. Natural products with skin-whitening effects. J. Food Drug Anal. 16 (2), 1-10.

Lionta, E., Spyrou, G., K Vassilatis, D., Cournia, Z., 2014. Structure-based virtual screening for drug discovery: principles, applications and recent advances. Curr. Top. Med. Chem. 14, 1923-1938.

Lipinski, C.A., Lombardo, F., Dominy, B.W., Feeney, P.J., 1997. Experimental and computational approaches to estimate solubility and permeability in drug discovery and development settings. Adv. Drug Deliv. Rev. 23, 3-25.

Masamoto, Y., Murata, Y., Baba, K., Shimoishi, Y., Tada, M., Takahata, K., 2004. Inhibitory effects of esculetin on melanin biosynthesis. Biol. Pharm. Bull. 27, 422-425.

Pillaiyar, T., Manickam, M., Namasivayam, V., 2017. Skin whitening agents: Medicinal chemistry perspective of tyrosinase inhibitors. J. Enzyme Inhib. Med. Chem. 32, 403-425.

Potts, R.O., Guy, R.H., 1992. Predicting skin permeability. Pharm. Res. 9, 663-669.

Reham S. Ibrahim, Rahma SR. Mahrous, Hoda M. Fathy , Abdallah A. Omar, and R.M.A.E.-K., 2020. Anticoagulant Activity Screening of an InHouse Database of Natural Compounds for Discovering Novel Selective Factor Xa Inhibitors; A Combined In-silico and In-Vitro Approach. Med. Chem. Res. https://doi.org/10.1007/s00044-02002516-5

Schrödinger, L.L.C., 2007. QikProp 3.0 User Manual.

Shakeri, A., Iranshahy, M., Iranshahi, M., 2014. Biological properties and molecular targets of umbelliprenin-a mini-review. J. Asian Nat. Prod. Res. 16, 884-889.

Shen, Jianhua, Xu, X., Cheng, F., Liu, H., Luo, X., Shen, Jingkang, Chen, K., Zhao, W., Shen, X., Jiang, H., 2003. Virtual screening on natural products for discovering active compounds and target information. Curr. Med. Chem. 10, 2327 2342.

Taddeo, V.A., Epifano, F., Preziuso, F., Fiorito, S., Caron, N., Rives, A., de Medina, P., Poirot, M., Silvente-Poirot, S., Genovese, S., 2019. HPLC Analysis and Skin Whitening Effects of Umbelliprenin-containing Extracts of Anethum Graveolens, Pimpinella Anisum, and Ferulago Campestris. Molecules 24, 501.

Taddeo, V.A., Genovese, S., de Medina, P., Palmisano, R., Epifano, F., Fiorito, S., 2017. Quantification of biologically active O-prenylated and unprenylated phenylpropanoids in dill (Anethum graveolens), anise (Pimpinella anisum), and wild celery (Angelica archangelica). J. Pharm. Biomed. Anal. 134, 319-324.

Zhou, H., Kepa, J.K., Siegel, D., Miura, S., Hiraki, Y., Ross, D., 2009. Benzene metabolite hydroquinone up-regulates chondromodulin-I and inhibits tube formation in human bone marrow endothelial cells. Mol. Pharmacol. 76, 579-587. 